\title{
Monte Carlo reservoir analysis combining seismic reflection data and informed priors
}

\author{
Zunino, Andrea; Mosegaard, Klaus; Lange, Katrine; Melnikova, Yulia; Hansen, Thomas Mejer
}

Published in:

Geophysics

Link to article, DOI:

10.1190/GEO2014-0052.1

Publication date:

2015

Document Version

Publisher's PDF, also known as Version of record

Link back to DTU Orbit

Citation (APA):

Zunino, A., Mosegaard, K., Lange, K., Melnikova, Y., \& Hansen, T. M. (2015). Monte Carlo reservoir analysis combining seismic reflection data and informed priors. Geophysics, 80(1), R31-R41.

https://doi.org/10.1190/GEO2014-0052.1

\section{General rights}

Copyright and moral rights for the publications made accessible in the public portal are retained by the authors and/or other copyright owners and it is a condition of accessing publications that users recognise and abide by the legal requirements associated with these rights.

- Users may download and print one copy of any publication from the public portal for the purpose of private study or research.

- You may not further distribute the material or use it for any profit-making activity or commercial gain

- You may freely distribute the URL identifying the publication in the public portal

If you believe that this document breaches copyright please contact us providing details, and we will remove access to the work immediately and investigate your claim. 


\title{
Monte Carlo reservoir analysis combining seismic reflection data and informed priors
}

\author{
Andrea Zunino ${ }^{1}$, Klaus Mosegaard ${ }^{1}$, Katrine Lange ${ }^{2}$, \\ Yulia Melnikova ${ }^{1}$, and Thomas Mejer Hansen ${ }^{1}$
}

\begin{abstract}
Determination of a petroleum reservoir structure and rock bulk properties relies extensively on inference from reflection seismology. However, classic deterministic methods to invert seismic data for reservoir properties suffer from some limitations, among which are the difficulty of handling complex, possibly nonlinear forward models, and the lack of robust uncertainty estimations. To overcome these limitations, we studied a methodology to invert seismic reflection data in the framework of the probabilistic approach to inverse problems, using a Markov chain Monte Carlo (McMC) algorithm with the goal to directly infer the rock facies and porosity of a target reservoir zone. We thus combined a rock-physics model with seismic data in a single inversion algorithm. For large data sets, the McMC method may become computationally impractical, so we relied on multiple-point-based a priori information to quantify geologically plausible models. We tested this methodology on a synthetic reservoir model. The solution of the inverse problem was then represented by a collection of facies and porosity reservoir models, which were samples of the posterior distribution. The final product included probability maps of the reservoir properties in obtained by performing statistical analysis on the collection of solutions.
\end{abstract}

\section{INTRODUCTION}

Inverse methods in geophysics are fundamental tools for unraveling the structure and rock properties within the subsurface of our planet. They are used to advance our knowledge in basic research and to provide crucial information in more applied contexts such as the oil and gas industry. In the latter case, understanding the structure and properties of the subsurface determines where to explore for hydrocarbon resources and how to effectively manage them. Seismic inversion has proven to be a key method to better understand the subsurface (e.g., Woodward et al., 2008; Bosch et al., 2010), returning high-resolution images. Nevertheless, in the context of exploration geophysics, inverting seismic data for only elastic properties is often not sufficient because the reservoir properties of interest are usually lithologies, fluid saturation, porosity, and other physical quantities.

Unfortunately, by using traditional methods, direct inference of reservoir properties is hampered by the difficulty of properly describing the link among seismic observables, elastic properties, and reservoir rock properties such as porosity or oil saturation, a relationship that can be described by rock-physics models. This inverse problem also suffers from the common pathology of nonuniqueness. Rock-physics models are often nonlinear, making common deterministic optimization methods not always suitable for performing direct inverse modeling for those properties. One common approach (e.g., Doyen, 2007) is a sequential procedure in which the seismic data are first inverted for elastic properties using a gradient-based or stochastic technique and then it transforms elastic properties into reservoir properties using rock-physics models and statistical analysis on well log data. This sequential scheme gives equivalent, but not identical, results to the joint approach in which elastic and reservoir properties are inverted simultaneously when all relationships are linear and Gaussian. On the contrary, if relationships are nonlinear, the sequential approach provides biased parameter estimation including maximum probability and mean (Bosch, 2004). Moreover, with this approach, it is not

\footnotetext{
Manuscript received by the Editor 3 February 2014; revised manuscript received 2 September 2014; published online 15 December 2014.

${ }^{1}$ Formerly Technical University of Denmark, Mathematical and Computational Geoscience, National Space Institute, and Center for Energy Resources Engineering, Lyngby, Denmark; presently Niels Bohr Institute, University of Copenhagen, Copenhagen, Denmark. E-mail: andrea.zunino@nbi.ku.dk; mosegaard@nbi.ku.dk; yume@space.dtu.dk; tmeha@space.dtu.dk.

${ }^{2}$ Technical University of Denmark, Mathematical and Computational Geoscience, National Space Institute, and Center for Energy Resources Engineering, Lyngby, Denmark. E-mail: katla@dtu.dk.

(C) 2014 Society of Exploration Geophysicists. All rights reserved.
} 
possible to obtain unbiased estimation of uncertainties; gradientbased methods linearize the problem around an initial solution, losing the information necessary for a correct uncertainty appraisal. Other approaches include Gaussian mixture models (Grana and Della Rossa, 2010) and prior models based on skewed distributions (Karimi et al., 2010). Uncertainty assessment is of utmost importance in inverse problems because the latter represents inferences from indirect measurements carrying uncertainties as well as an approximated description of the physics of the system, i.e., the forward model (Gouveia and Scales, 1998; Tarantola, 2005). Specifically, for the problem described in this paper, in which the prior model can be expressed only by generating samples from numerical procedures, sampling methods represent a more effective strategy to correctly estimate uncertainties (Mosegaard, 1998).

The joint approach using Monte Carlo methods based on a probabilistic framework provides some advantages. Monte Carlo methods can handle the nonlinearities of the forward model (e.g., rockphysics models) because only forward modeling and sampling of the prior distribution are needed to run the algorithm, i.e., there is no need to compute derivatives, unless an invariant definition of conditional probability is pursued (Mosegaard and Tarantola, 2002). The algorithm explores the model space extensively, so that, theoretically, several regions of high posterior probability may be sampled, resulting in a more comprehensive characterization of possible solutions. It is also easy to include hard/soft constraints in the algorithm such as well-log data because it only requires a routine able to generate models according to the prior information. Finally, a more robust estimation of uncertainties is provided by the collection of solutions sampled by the algorithm. However, this methodology is computationally very demanding; hence, some techniques to increase the efficiency are needed. In general, all problem-independent algorithms (metaheuristics) perform the same when averaged over all conceivable problems (the so-called blind search) (Mosegaard, 2012), but tailoring an algorithm for a specific problem may increase efficiency.

Using informed priors, i.e., priors that carry a significant amount of information relying on expertise or independent data, through the incorporation of geostatistics, has become commonplace in reservoir characterization, using multipoint statistics (e.g., Hansen et al., 2008; Cordua et al., 2012; Hansen et al., 2012) and Markov models for facies classification (Ulvmoen and Omre, 2010; Ulvmoen et al., 2010). Several attempts have been made to invert for reservoir properties that integrate seismic inversion with rock physics and geostatistics; a review can be found in Bosch et al. (2010). Some are deterministic inversions, which result in a single solution (Bosch, 2004), and some are fully (Bosch et al., 2007) or partially stochastic (González et al., 2008). Most of this work relies on a combination of traditional seismic inversion and geostatistics to take into account spatial correlations of facies and reservoir properties. Geostatistics can be represented by the two-point statistic of variograms (e.g., Journel and Huijbregts, 1978; Grana et al., 2012) or by the more advanced multipoint statistics method that is able to capture patterns from training images (e.g., Strebelle, 2002; González et al., 2008; Remy et al., 2009). The strategy we propose in this paper is to use informed priors to introduce a priori information into the algorithm, reducing the size of the search space, thus increasing efficiency (Cordua et al., 2012; Hansen et al., 2012). We use some form of complex geologic prior knowledge, which helps restrict the number of possible models that are proposed by the algorithm.
We provide a study in which we combine (1) a rock-physics model, (2) a complex prior based on multiple point statistics, and (3) reflection seismic data whereby, unlike previous studies so far in which some approximations were in place, we are able to obtain actual samples from the posterior using a "pure" probabilistic approach, i.e., strictly adhering to the theory explained in Mosegaard and Tarantola (2002).

Our aim is to develop an algorithm that is capable of inverting relatively large data sets combining geostatistics, rock physics, and seismology for a 3D arrangement of voxels representing physical properties. We illustrate the results of the inversion of seismic reflection data from a synthetic test model showing how it is possible to invert directly for reservoir properties in a probabilistic framework. Using techniques derived from geostatistics, we are able to convey prior information that helps to make the computational problem tractable by reducing the space of possible models. We also provide uncertainty assessment discussing some marginals on porosity and facies. The application of this methodology using a real data set of measurements will be the subject of another paper.

\section{PROBABILISTIC INVERSE RESERVOIR MODELING}

To understand structure and properties of the subsurface, we rely on indirect measurements mostly performed on the surface of the earth using an approximate description of the physical system. The measurements are affected by uncertainties that must be taken into account. For this reason, we use methods drawn from probabilistic inverse theory to infer the sought properties.

Monte Carlo sampling methods provide a solution to the inverse problem in the form of a collection of models that approximate the posterior distribution. In our case, we are interested in the "importance sampling" methods because of their particular properties. Sample points of a probability distribution are defined as points such that the probability of any point to be inside a certain domain equals the probability of the domain (Tarantola, 2005). Basically, using these techniques, the solutions are sampled at a rate proportional to their a posteriori probability (importance sampling); i.e., models that better fit the observed data and are consistent with the a priori information are sampled more frequently. This ensemble of models can be analyzed to find out a particular feature of the models in that this collection encloses all the information we have on the posterior. If, for instance, we are interested in the porosity in a particular area of the model, we can make a histogram of the porosity in that area using the collection of solution models and review the possible scenarios. Sampling the posterior helps us to understand whether we are in the presence of, e.g., marginal Gaussian distributions, for which we might be interested in computing the mean model, its variance, or in more complex, multimodal distributions, that give rise to a multitude of quite different but plausible scenarios.

In this framework, three ingredients are then needed for a comprehensive investigation of reservoir properties: (1) An algorithm that uses geostatistics to generate realizations of the prior models, (2) a rock-physics model linking bulk rock properties with elastic parameters, and (3) a technique to compute seismograms from elastic properties at given locations on the surface. Our aim is to combine these ingredients in a simple, consistent, and comprehensive algorithm to sample the posterior distribution. 


\section{A glimpse of the Markov chain Monte Carlo method for reservoir model analysis}

\section{Background theory}

The general solution to inverse problems in the probabilistic approach is given by Mosegaard and Tarantola (2002) and Tarantola (2005)

$$
\sigma(\mathbf{d}, \mathbf{m})=k \frac{\rho(\mathbf{d}, \mathbf{m}) \Theta(\mathbf{d}, \mathbf{m})}{\mu(\mathbf{d}, \mathbf{m})}
$$

where the posterior distribution $\sigma(\mathbf{d}, \mathbf{m})$ is computed from a normalization constant $k$, the homogeneous state of information $\mu(\mathbf{d}, \mathbf{m})$ and the combination of the prior $\rho(\mathbf{d}, \mathbf{m})$ and theoretical $\Theta(\mathbf{d}, \mathbf{m})$ probability densities. Note that $\mathbf{d}$ and $\mathbf{m}$ belong to data $D$ and model $M$ space, respectively. However, we are interested in the marginal $\sigma_{M}(\mathbf{m})$, which represents the posterior probability in the model space, telling us the probabilities associated with all conceivable models. Under the assumption that $\mu(\mathbf{d}, \mathbf{m})=\mu(\mathbf{d}) \mu(\mathbf{m})$, this marginal is given by

$$
\begin{aligned}
\sigma_{M}(\mathbf{m}) & =\int_{D} k \frac{\rho(\mathbf{d}, \mathbf{m}) \Theta(\mathbf{d}, \mathbf{m})}{\mu(\mathbf{d}, \mathbf{m})} \mathrm{d} \mathbf{d} \\
& =k \rho_{M}(\mathbf{m}) \int_{D} \frac{\rho_{D}(\mathbf{d}) \Theta(\mathbf{d} \mid \mathbf{m})}{\mu_{D}(\mathbf{d})} \mathrm{d} \mathbf{d} \\
& =k \rho_{M}(\mathbf{m}) L(\mathbf{m}) .
\end{aligned}
$$

The two densities $\rho_{M}(\mathbf{m})$ and $L(\mathbf{m})$ represent the prior and the likelihood function that measures the degree of fit between the calculated and the measured data, respectively. The objective of our inverse problem is then to characterize the posterior distribution.

Mosegaard and Tarantola (1995) propose an algorithm (called the extended Metropolis algorithm in Mosegaard, 2006), which is able to sample the posterior distribution given a prior model generator and a routine able to perform the forward modeling. These are the only two necessary elements to perform sampling of solutions to the inverse problem. The value of the prior distribution does not need to be known exactly. This is a Markov chain Monte Carlo (McMC) method because it is based on a Markov process; i.e., the next state of the chain depends only on the current state (no memory).

\section{Inversion algorithm}

The extended Metropolis sampler is shown in Algorithm 1 as a pseudocode. We first initialize the two variables niter and nsave, which represent the requested number of iterations and the number of proposed models to skip before saving the current model in the ensemble of solutions. The Metropolis algorithm produces realizations that are correlated; therefore, it is necessary to discard part of them, i.e., save a model in the collection of solutions only every nsave proposed models, to obtain statistically independent models. This variable should be estimated after some trial runs, analyzing the correlation of models to ensure independence of samples. This operation could be carried out after a complete run of the algorithm, namely, after the collection of samples has been obtained. However, the storage requirements for a high number of iterations and large 3D models may prevent the application of the latter procedure, as discussed in the numerical experiments described below. The next step is to set an arbitrary starting model as the "current" model $\mathbf{m}_{\text {cur }}$ (Algorithm 1, line 2). Theoretically, the influence of this starting model on the performance of the algorithm is only on the length of the burn-in phase, i.e., the first stage, when the algorithm tries to locate a high-probability region to start sampling. Practically, if the number of iterations that is run afterward is insufficient, the algorithm may fail to explore some high-probability regions depending on the starting model. The closer the starting model is to a high-probability region, the shorter is the burn-in phase. After setting the starting model, the iterative part of the algorithm starts ("for" loop in Algorithm 1, line 3), which will continue for niter iterations. First, a new model is drawn from the prior distribution; i.e., a new model that is in agreement with the prior information is proposed. In practice, the current model is somehow modified or "perturbed" to obtain the new model $\mathbf{m}_{\text {new }}$, for instance, randomly changing only a small volume of it. The new proposed model $\mathbf{m}_{\text {new }}$ is accepted as the next move of the random walk with probability $P_{\text {acc }}$ following the Metropolis rule (Mosegaard and Tarantola, 1995) (Algorithm 1 line 5). Notice that, when using this rule, the normalization constant $k$ of equation 2 does not need to be calculated explicitly because it appears on the numerator and denominator of the relation shown on line 5 of Algorithm 1 and is hence simplified. If accepted, the new proposed model becomes the current model (Algorithm 1, line 7); otherwise, the random walk stays at the current position (Algorithm 1, line 9). Finally, the model is retained in the ensemble of solutions only every nsave proposed models (Algorithm 1, lines 11 and 12, in which "mod" indicates the modulo).

Importance sampling is a useful property of this method because models with higher posterior probability are sampled more frequently by the algorithm, so that volumes of high probability in the posterior distribution are characterized by a denser sampling. This allows us to use statistical inference once we obtain the collection of solutions. We can then calculate any kind of information $\Phi(\mathbf{m})$ related to the probability $\sigma(\mathbf{m})$ using the following relationship:

$$
\int_{M} \Phi(\mathbf{m}) \sigma(\mathbf{m}) \mathrm{d} \mathbf{m} \approx \frac{1}{N} \sum_{i=1}^{N} \Phi\left(\mathbf{m}^{(i)}\right),
$$

\section{Algorithm 1. Extended Metropolis sampler.}

1: set niter, nsave

2: select a starting model and set it as the current model $\mathbf{m}_{\text {cur }}$

3: for $i=1$, niter do

4: perform one step with the prior sampler to obtain $\mathbf{m}_{\text {new }}$

5: $\quad \mathbf{m}_{\text {new }}$ is accepted with probability

$$
P_{\mathrm{acc}}=\min \left[1, \frac{L\left(\mathbf{m}_{\mathrm{new}}\right)}{L\left(\mathbf{m}_{\mathrm{cur}}\right)}\right]
$$

6: if $\mathbf{m}_{\text {new }}$ is accepted then

7: $\quad \mathbf{m}_{\text {cur }}=\mathbf{m}_{\text {new }}$

8: $\quad$ else

9: $\quad \mathbf{m}_{\mathrm{cur}}=\mathbf{m}_{\mathrm{cur}}$

10: end if

11: $\quad$ if $i(\bmod n s a v e)==0$ then

12: $\quad$ save $\mathbf{m}_{\text {cur }}$ in the collection of solutions

13: end if

14: end for 
where $N$ is the number of models sampled (the ensemble of solutions) and $\mathbf{m}^{(i)}$ represents a series of independent draws. The error of the integral tends toward zero with growing $N$. Some examples of the use of this formula are shown in the result of the numerical experiments.

To improve the performance of the Metropolis algorithm, we exploit a parallel implementation that significantly decreases the run time. Instead of computing one forward model at a time, we concurrently draw several perturbed models from the prior and compute in parallel all likelihoods, a technique similarly proposed by Brockwell (2006). This "prefetching" technique is computationally more costly because more models are unused than in the traditional Metropolis, but it takes advantage of parallelization, saving a significant amount of run time. The prefetching Metropolis sampler is shown in Algorithm 2.

The main difference compared to Algorithm 1 is that many new models, depending on the number of CPUs used (ncpus), is proposed from the current model at each iteration. The computation of the likelihood for these models is then carried out in parallel, saving a significant amount of time that depends also on the rate of acceptance for the particular run.

\section{Algorithm 2. Extended Metropolis sampler.}

1: set niter, nsave and ncpus

2: $n$ prmod $=0$

3: select a starting model and set it as the current model $\mathbf{m}_{\text {cur }}$

4: for $i=1$, niter do

5: spread the current model to all CPUs, so that $\mathbf{m}_{\mathrm{cur}}^{(p=1, \ldots, n c p u s)}=\mathbf{m}_{\mathrm{cur}}$

6: for $p=1$, ncpus [IN PARALLEL] do

7: $\quad$ perform one step with the prior sampler to obtain $\mathbf{m}_{\text {new }}^{(p)}$

8: $\quad$ compute the likelihood $L\left(\mathbf{m}_{\text {new }}^{(p)}\right.$

9: end for

10: for $p=1$, ncpus do

11: $\quad$ nprmod $=$ nprmod +1

12: $\quad \mathbf{m}_{\text {new }}^{(p)}$ is accepted with probability

$$
P_{\mathrm{acc}}^{(p)}=\min \left[1, \frac{L\left(\mathbf{m}_{\mathrm{new}}^{(p)}\right)}{L\left(\mathbf{m}_{\mathrm{cur}}\right)}\right]
$$

13: $\quad$ if $\mathbf{m}_{\text {new }}^{(p)}$ is accepted then

14: $\quad \mathbf{m}_{\text {cur }}=\mathbf{m}_{\text {new }}^{(p)}$

15: $\quad$ if nprmod $(\bmod n s a v e)==0$ then

16: save $\mathbf{m}_{\text {cur }}$ in the collection of solutions

17: $\quad$ end if

18: $\quad$ exit from inner loop

19: $\quad$ else

20: $\quad \mathbf{m}_{\text {cur }}=\mathbf{m}_{\text {cur }}^{(p)}$

21: $\quad$ end if

22: $\quad$ if nprmod $(\bmod n s a v e)==0$ then

23: $\quad$ save $\mathbf{m}_{\text {cur }}$ in the collection of solutions

24: $\quad$ end if

25: end for

26: end for
Our algorithm follows the requirements described in Mosegaard and Tarantola (1995), and, therefore, it samples the posterior distribution. We construct a multistep algorithm, including a rockphysics model, seismic modeling, and geostatistics, capable of sampling the posterior distribution that is described in the following. We finally obtain a collection of solutions in the model space $M$.

\section{Geostatistical prior model}

High-dimensional spaces tend to be empty, suffering from the socalled curse of dimensionality (Tarantola, 2005); therefore, sampling them is a difficult task that usually requires a tremendous computational effort. Because of the large number of model parameters, the size of the model space to be searched is often so huge that the computational time needed simply cannot be afforded. In this case, the most effective way of addressing this problem is to use all the available prior knowledge and convey it into the sampling algorithm so that the number of possible models to be sampled is greatly reduced. If the prior model has significant spatial correlations, as it is assumed for subsurface properties, the effective number of degrees of freedom is usually smaller than the number of parameters. This may help in increasing the efficiency (Hansen et al., 2009).

A noninformed sampling for reservoir properties is not feasible for even a subset of the number of parameters necessary in a real application because of the huge number of iterations it would require. To overcome this difficulty, we propose to use all available prior information to constrain the solution and reduce the size of the space of possible models. A traditional way of inputting of prior information has been to use the distance from some given model (perhaps under Gaussian assumptions), which in practice is often difficult to provide. Recently, a more sophisticated technique has appeared to input complex a priori information into probabilistic inverse problems (González et al., 2008; Cordua et al., 2012; Hansen et al., 2012). This technique relies on geostatistics to generate realizations of prior models taking into account multiple-point statistics by means of the occurrence of patterns in a given model. Specifically, the geostatistical algorithm uses prototype models, which embed the structure of the subsurface expected in the area of study, in the form of training images (Guardiano and Srivastava, 1993; Strebelle, 2002) to generate new realizations of the model such that they hold the same statistical properties, in terms of patterns, as the training image. These training images are a means to translate the "expert" knowledge (information) from, for instance, a geologist into the inversion algorithm.

The multipoint algorithm is thus used to generate realizations of the reservoir model according to the prior knowledge, the first step in the proposed McMC method. However, generating an entirely new model at each iteration of the random walk would be too large of a step for the Metropolis sampler, resulting in a very low acceptance ratio. Therefore, the size of the volume that is resimulated at each iteration needs to be small compared to the size of the entire model. In this way, the steps performed by the random walk are similarly small. The location and size of the volume to be resimulated are selected randomly at each iteration. This is an application of the sequential Gibbs sampler as described by Hansen et al. (2012). Moreover, in approximately $10 \%$ of the cases, the algorithm attempts to perform a bigger step to encourage the random walk to explore other places of the posterior distribution, aiming at improving mixing. The size of the patterns and the volume to be resimulated determine the amount of structure (or correlations) that we 
convey in the algorithm (line 4 in Algorithm 1), affecting the number of iterations needed to converge toward the equilibrium probability and also restricting the model space.

It is important to remark that the input prior information must be carefully studied, as its weight on the solution to the inverse problem might be high. Wrong prior information (geologic scenario, prototype model) may result in a biased posterior assessment and could potentially drive the interpretation away from the real solution. Therefore, a careful critique must be applied when deciding the amount and type of information to be introduced in the inversion process.

\section{NUMERICAL EXPERIMENTS}

In this section, we illustrate the application of the methodology using a numerical example. We construct a synthetic set of seismic measurements using a 3D synthetic reservoir model. The observed data $\mathbf{d}_{\text {obs }}$ are seismograms obtained convolving the reflection coefficients obtained from the model with a $50-\mathrm{Hz}$ Ricker wavelet, as explained below, simulating a data set from a seismic survey processed to obtain zero-offset equivalent data. Correlated, zero-mean Gaussian noise with a standard deviation of 0.14 and a given covariance matrix is added to the observed data to simulate measurement errors. Our target properties are facies and porosity, i.e., model parameters to be derived. Hard constraints, such as data from wells, can easily be handled by this algorithm, but we consider all properties sought as unknown at all locations in our model. To sample the posterior distribution, we set up a McMC algorithm following Algorithm 2 described above. It is composed of three main elements: a prior generator, forward modeling routines including rock physics and seismic modeling, and the extended Metropolis sampler. The prior sampler produces facies and porosity models according to our prior information.

In the following, we describe the parameterization of our problem, the procedure to sample the posterior, all elements that form the forward model, and finally we discuss the results.

\section{Parameterization}

We construct a synthetic example derived from the Stanford VI reservoir model (Lee and Mukerji, 2012), using only the upper layer. The Stanford model represents a fluvial channel system and is comprised of three different layers, representing deltaic deposits, meandering channels, and sinuous channels, the latter used in this study. We preserve the geometry of the channels and use the same rock-physics model, but we reassign all properties. We upscale the upper layer of the model and considered only two facies: shale and sand. The number of voxels in the $x$-, $y$-, and $z$-directions is 38,50 , and 20 , with dimensions of 100,100 , and $4 \mathrm{~m}$, respectively. This model ideally represents a target zone of a larger reservoir. Figure 1 depicts the reservoir model used to compute the synthetic measurements that constitute the "observed data" (Figure 2) of our problem.

\section{Sampling the prior}

First, we use an algorithm that uses geostatistics to generate facies models from the prior distribution. This algorithm, given a current model of facies, is capable of generating new realizations of the model using the information retrieved from a training image. To generate new realizations of the reservoir model, the new model, we perturb the current facies model following the procedure described in Lange et al. (2012), in which only a block (a subset) of the 3D model is changed using sequential simulation. The template is a 3D hyper-rectangle that defines the geometry and size of the patterns that we are looking for. Given a particular voxel in the model, the template is defined by its surrounding voxels (Lange et al., 2012). We choose a small template with dimensions of (3, 3,3 ) voxels. Figure 3 shows the training image. As explained above, generating a completely new realization of a model results in a significant step in the random walk, so we choose to resimulate only a small volume of the model at a time. This volume has a randomly variable size (which corresponds to a variable step length in the McMC algorithm), defined for each direction $x, y$ within the range $[3,5]$, and 3 voxels for the $z$-direction with a $90 \%$ probability for the normal step and $[5,7]$ for $x, y$, and $[3,5]$ voxels for the $z$-direction with the remaining $10 \%$ probability for the long step. Because the probability to move from the current state to the new and vice versa is the same, the property of detailed balance is still satisfied; therefore, the chain will equilibrate at the correct equilibrium distribution.

The second step is to compute the porosity of each voxel describing it as a random variable linked to the facies model. Because porosity $\phi$ is bounded between $[0,1]$, following Bosch et al.

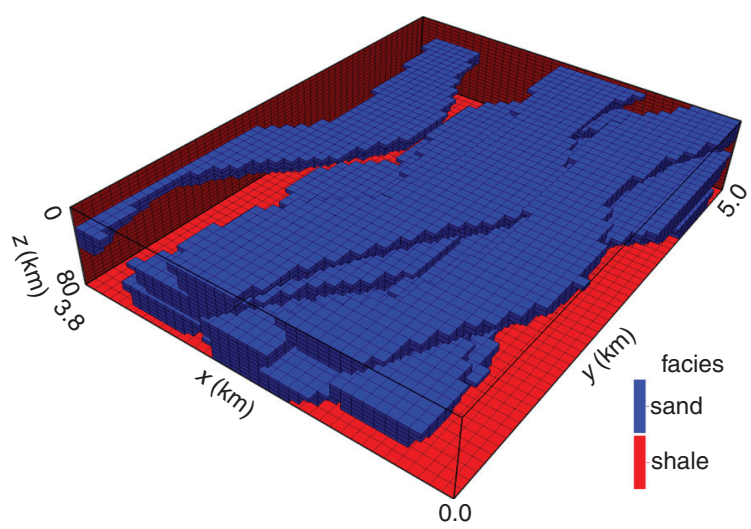

Figure 1. The two-facies reservoir model used in the numerical experiments to compute the synthetic measured data. Red voxels represent shale, whereas the blue represents sand.

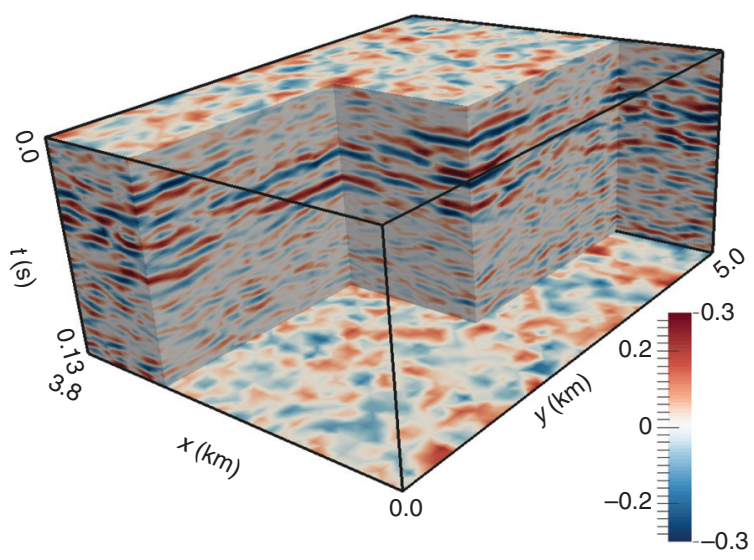

Figure 2. Plot of synthetic observed data showing slices through the seismic volume. 
(2007), we apply a transformation to logarithmic porosity that allows for the use of Gaussian modeling. The logarithmic porosity $\phi^{*}$ and its back-transform are then given by

$$
\phi^{*}=\ln \left[\frac{\phi}{(1-\phi)}\right] \text { and } \phi=\frac{\exp \left(\phi^{*}\right)}{\left(1+\exp \left(\phi^{*}\right)\right)} .
$$

We then model the logarithmic porosity as a random variable using a Gaussian density function, with mean and variance depending on the facies. In the reference model, porosity has a mean value of 0.3 and 0.05 for sand and shale, respectively.

Our model $\mathbf{m}$ is now composed by two arrays: facies and porosity. In this setup, the prior distribution of facies is not known explicitly, but we are able to sample it; whereas for porosity, the distribution is known. For simplicity, in this study, porosity is assumed to be spatially independent.

\section{Solving the forward problem}

We now describe in detail all the steps involved in calculating the synthetic data $\mathbf{d}_{\text {calc }}=g(\mathbf{m})$ using the nonlinear forward model $g(\mathbf{m})$, where $g$ represents the mapping between model parameters and calculated seismic data.

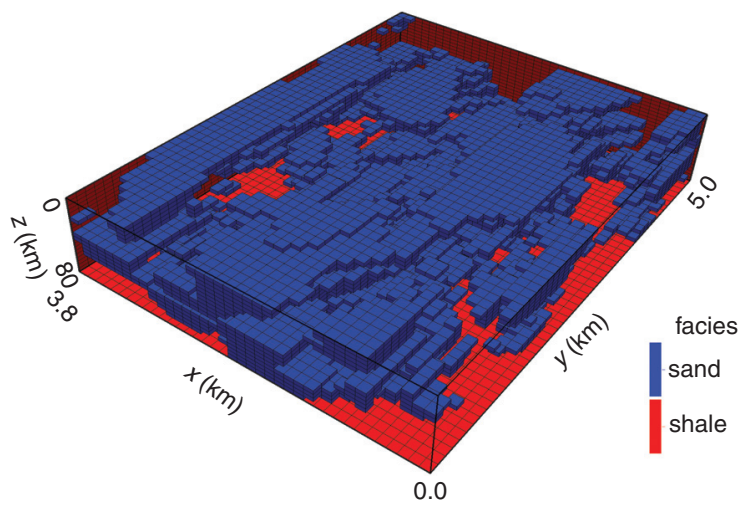

Figure 3. The training image used by the multipoint geostatistical algorithm.

Table 1. Elastic properties and density of minerals and fluids.

Sand Shale $\rho(\mathrm{g} / \mathrm{cc}) K(\mathrm{GPa}) G(\mathrm{GPa})$ vol. frac. vol. frac.

\begin{tabular}{lrrrrr}
\hline Minerals & & & & & \\
Clay & 2.50 & 21.0 & 9.0 & 0.00 & 0.85 \\
Quartz & 2.65 & 36.6 & 44.0 & 0.65 & 0.15 \\
Feldspar & 2.63 & 75.6 & 25.6 & 0.20 & 0.00 \\
Rock fragments & 2.70 & 80.0 & 20.0 & 0.15 & 0.00 \\
Fluids & & & & & \\
Brine & 0.99 & 2.57 & - & - & - \\
Oil & 0.70 & 0.50 & - & - & - \\
\hline
\end{tabular}

\section{Rock physics}

The rock-physics model is a fundamental element that links the bulk rock properties (in our case, facies and porosity) and physical conditions with elastic parameters. We follow the Stanford VI reservoir model (Lee and Mukerji, 2012). We consider the rocks to be composed of three minerals, clay, quartz, and feldspar, and rock fragments with known volume fraction. In a real case, volume fractions are estimated with a possibly significant uncertainty, which should be taken into account in the algorithm; however, for simplicity, to keep the computational cost feasible, we assume to know their value. We model two different facies: shale and sand. The sand facies is oil-/brine-saturated (oil saturation equal to 0.85), whereas the shale facies is fully brine saturated. The sand facies is considered a poorly cemented sandstone. The objective is to compute the elastic bulk and shear moduli $K, G$, and the density $\rho$ of the bulk rock. In our model, these parameters depend on facies and porosity. The elastic properties and density of minerals and fluids used in this experiment are summarized in Table 1.

When brine and oil are present, it is possible to compute the effective density using the concept of the effective fluid as

$$
\rho_{f}=\sum_{i=1}^{2} S_{i} \rho_{f(i)}
$$

where $\rho_{f(i)}$ is the density of the single fluid $i$ and $S_{i}$ is the saturation of the fluid $i$. The density of the bulk rock can be computed as a volumetric average of the densities of the minerals as

$$
\rho=\phi \rho_{f}+(1-\phi) \sum_{i=1}^{N} V_{m(i)} \rho_{m(i)}
$$

where $i$ represents the mineral constituents and $V_{m}$ and $\rho_{m}$ are their volume fraction and density, respectively.

\section{Sand facies}

We compute the bulk and shear modulus of the solid phase $K_{s}$ and $G_{s}$ using the Voigt-Reuss-Hill average (Hill, 1952) as

$$
X_{V}=\sum_{i=1}^{N} f_{i} M_{i} ; X_{R}=\left(\sum_{i=1}^{N} \frac{f_{i}}{M_{i}}\right)^{-1} ; \quad X_{V R H}=\frac{X_{V}+X_{R}}{2} \text {, }
$$

where $X$ is to be substituted by $K_{s}$ and $G_{s}, f_{i}$ is the volume fraction of the mineral; and $M_{i}$ is the elastic modulus of the mineral.

We assume that the sand facies is characterized by a constant amount of contact cement, so that porosity varies only due to noncontact pore-filling material, that is, poorly sorted (Avseth et al., 2000). The rock-physics model for this scenario is the "constant cement theory" (Avseth et al., 2000). This model requires the need to compute the moduli $K_{b}$ and $G_{b}$ at the initial sand pack porosity $\phi_{b}$ derived from contact cement deposition from contact cement theory (Dvorkin and Nur, 1996). Contact cement theory and various parameters involved in the rock-physics computations are described in Appendix A. The theoretical background and detailed descriptions of rock-physics models can be found in Avseth et al. (2005), Mavko et al. (2009), and Dvorkin and Gutierrez (2014). 
We compute $K_{\text {dry }}$ and $G_{\text {dry }}$ from constant cement theory (Avseth et al., 2000):

$\begin{aligned} K_{\mathrm{dry}} & =\left(\frac{\phi / \phi_{b}}{K_{b}+4 G_{b} / 3}+\frac{1-\phi / \phi_{b}}{K_{s}+4 G_{b} / 3}\right)^{-1}-\frac{4 G_{b}}{3} ; \\ G_{\mathrm{dry}} & =\left(\frac{\phi / \phi_{b}}{G_{b}+z}+\frac{1-\phi / \phi_{b}}{G_{s}+z}\right)^{-1}-z\end{aligned}$

and

$$
z=\frac{G_{b}}{6} \frac{9 K_{b}+8 G_{b}}{K_{b}+2 G_{b}}
$$

The next step is to compute the elastic moduli for the saturated rock $K_{\text {sat }}$ and $G_{\text {sat }}$ using Gassmann's equation (Gassmann, 1951):

$$
K_{\mathrm{sat}}=K_{s}\left[\frac{\phi K_{\mathrm{dry}}-(1+\phi) K_{f} \frac{K_{\mathrm{dry}}}{K_{s}}+K_{f}}{(1-\phi) K_{f}+\phi K_{s}-K_{f} \frac{K_{\mathrm{dry}}}{K_{s}}}\right]
$$

and

$$
G_{\text {sat }}=G_{\text {dry }} .
$$

Finally, the isotropic $V_{\mathrm{P}}$ formula is used as

$$
V_{\mathrm{P}}=\sqrt{\frac{K+\frac{4}{3} G}{\rho}}
$$

\section{Shale facies}

The P-wave velocity for shale facies is modeled by using Gardner's relationship (Gardner et al., 1974) as defined for the Stanford VI model:

$$
V_{\mathrm{P}}=\left(\frac{\rho}{d}\right)^{\frac{1}{a}}, \quad d=1.75, \quad a=0.265
$$

where $\rho$ is expressed in grams per cubic centimeter.

\section{Seismic modeling}

We examine zero-offset seismograms measured on top of each column of voxels, for a total number of 1900 traces. To compute the seismograms $u(t)$, we convolve the reflection coefficient series with a 50-Hz Ricker wavelet:

$$
u(t)=R(t) * W(t),
$$

where $R(t)$ is the reflection series in time $t$ and $W(t)$ is the wavelet. These computations are carried out in the frequency domain exploiting the Hermitian symmetry for the Fourier transform of a real-valued input signal. The time-dependent series of reflection coefficients is computed first using the velocity and density model to obtain impedance, then a conversion from depth to time and finally computation of the reflection coefficients. For simplicity, the wavelet is assumed to be known, which is often not the case in practice. In the latter case, the uncertainty on the wavelet can, in principle, be included in the algorithm.

\section{Sampling the posterior}

The next step in the extended Metropolis algorithm is to define a likelihood function. We model the measurement errors as having a Gaussian distribution; hence, given a nonlinear forward model $g(\mathbf{m})$, we arrive at a likelihood function in the form of the following equation:

$$
L(\mathbf{m})=k \exp \left[-\frac{1}{2}\left(g(\mathbf{m})-\mathbf{d}_{o b s}\right)^{T} \mathbf{C}_{D}^{-1}\left(g(\mathbf{m})-\mathbf{d}_{o b s}\right)\right],
$$

where $\mathbf{C}_{\mathbf{D}}=\mathbf{C}_{\mathbf{d}}+\mathbf{C}_{\mathbf{T}}$ represents the covariance of the data $\mathbf{C}_{\mathbf{d}}$ plus the uncertainty associated with the forward modelization $\mathbf{C}_{\mathbf{T}}$ because we assume both of them to be Gaussian distributed (Tarantola, 2005).

Finally, the posterior distribution is sampled by running Algorithm 2, using samples from the prior as input and performing the forward calculations to obtain the likelihood function and use it in the Metropolis sampler.

\section{Results and discussion}

Using the method described above, we ran Algorithm 2 to propose a total of $50 \times 10^{6}$ models to the Metropolis sampler. We retain only one model out of every 100 in the collection of solutions to ensure independence. This last value can be obtained using correlation between realizations. Figure 4 shows the facies starting model used in this numerical study. Figure 5 depicts likelihood as a function of the number of proposed models. The porosity mean values for the inversion are 0.25 and 0.07 for sand and shale, respectively, different from the reference model to simulate some uncertainty. The so-called burn-in phase, a typical feature of McMC algorithms, is clearly visible, spanning approximately $25 \times 10^{6}$ iterations. In this random-walk phase, beginning from the initial model, the program searches for regions of high probability in model space. The models resulting from burn-in are thus discarded and not part of the solution of the inverse problem. This run took approximately six days on a $2.6-\mathrm{GHz}$ six-core desktop computer. The amount of memory used is quite limited, in this case being approximately $50 \mathrm{MB}$ per processor.

We end up with a collection of models representing samples of the posterior distribution that can be used to estimate subsurface

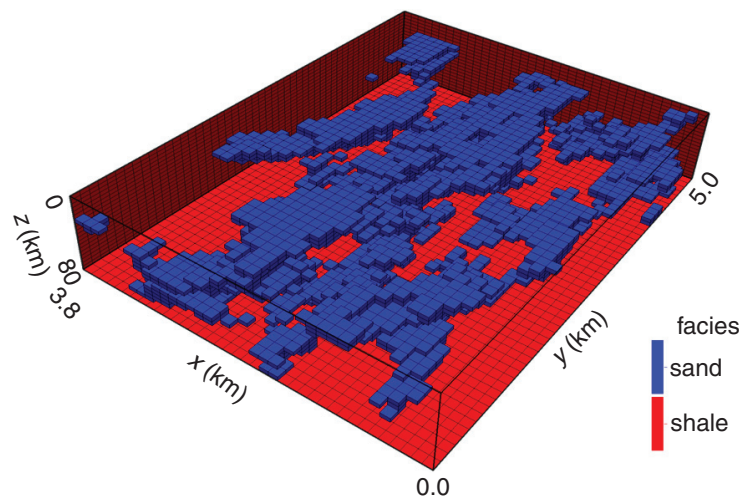

Figure 4. The starting model used to initialize the McMC algorithm in the numerical study. 
properties and their relative probabilities/uncertainties. Every posterior ensemble model represents a solution of the inverse problem, being consistent with the prior information and fitting the measured data. Figure 6 depicts randomly picked facies models. Once we

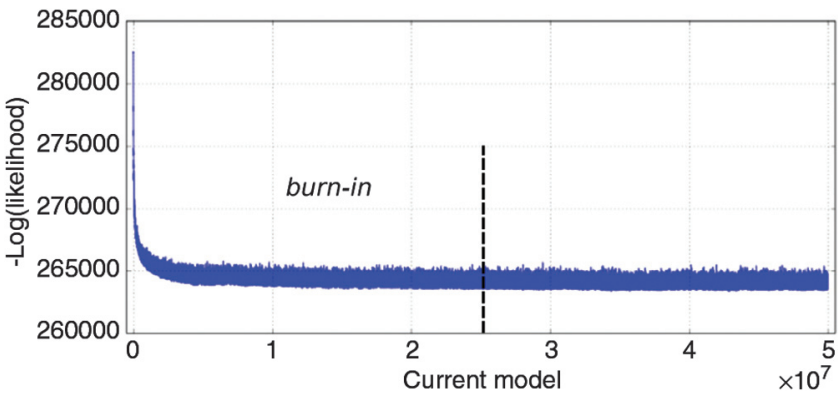

Figure 5. Negative logarithm of likelihood as a function of the number of current model. obtain the ensemble of solutions, we can use equation 3 to query the database about several different aspects because it represents a complete inverse problem solution.

The uncertainty of results is affected by several factors such as noise in the data, the accuracy of the forward model and, in the case of multipoint statistics, the uncertainty related to the training image. The training image represents a technique to describe the geologic knowledge of the area under study that, in real cases, can be insufficient. This may result in wrong assessment of the structures in the subsurface therefore biasing the inversion process. Because of this reason, careful study of the area is needed when using training images. Nevertheless, similar issues concerning uncertainty arise also when more classical Gaussian priors are used. In our numerical experiment, the training image is not optimal because the structures are only partially similar to those of the reference model to simulate a real case.

We now discuss a few examples of the kind of information that is possible to retrieve. The first target is porosity. We calculate the average model of porosity using 250,000 models from the posterior collection (Figure 7). Overall, this model is in good agreement with the reference one. However, in some specific areas, it is not matching the reference value. This uncertainty can be due to the effect of the noise on the data; i.e., different models fit equally well the observed data and thus posterior porosity distribution may result in being multimodal. In our numerical experiment, the spatial correlation of porosity is determined by the spatial occurrence of facies because the mean of the distribution of logarithmic porosity depends on facies, producing sharp changes of porosity at the transition from one facies to another one. This limitation is necessary to restrict the computational cost; however, in principle, it would be possible to include a more sophisticated approach to handle spatial correlation of porosity. We compute the value of porosity at two particular locations, one at $(x, y, z)=$ $(1500,3500,20) \mathrm{m}$ and the other at $(200,200$, 0 ) m, obtaining a histogram of porosity (Figure 8). The histogram provides the ranges of possible values and their probability. The red histogram in

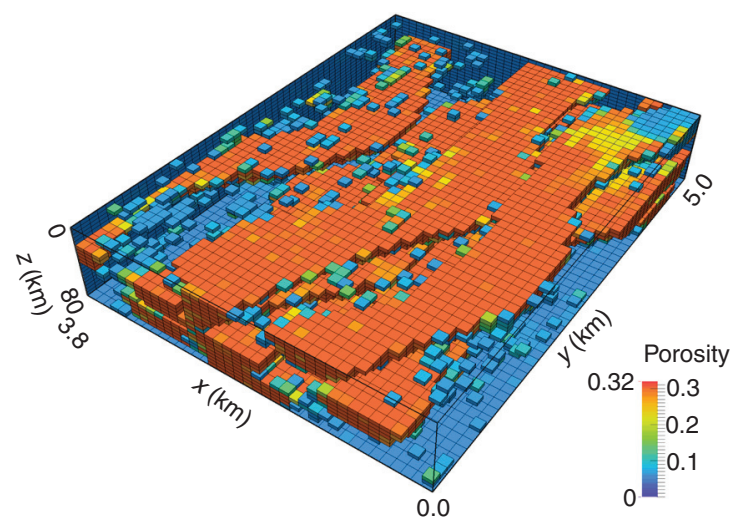

Figure 7 . The average porosity model computed using the posterior collection of models. The values inside the volume with a value lower than 0.1 are not shown to allow us to see through the volume.
Figure 8 shows a prominent peak approximately 0.30 . The peak indicates that porosity in that particular location is well resolved, with a very low probability between 0.21 and 0.28 , indicating a small degree of uncertainty. The values spanned by this histogram suggest we are in the presence of a sand facies because the mode value of porosity is approximately 0.30 . The blue histogram in Figure 8, on the other hand, is significantly multimodal, very narrow, and peaked around 0.05 with a secondary peak at approximately 0.07 , indicating a more uncertain estimation of porosity at that location. In this case, the range of porosity values spanned suggests we are in a shale facies.

Another example of information inferred from the posterior collection of models is the computation of the probability to have sand, equivalent to channels in our simplified model, in the 3D volume of the reservoir model. Figure 9 shows a representation of the probability of having sand seen from above and below, and it also depicts the morphology of channels as derived by the ensemble of solutions. Because facies is a categorical variable, it is possible 
to calculate a facies probability map, for instance, focusing on sand as in our example, by counting the number of occurrences of each facies in each voxel for every model and dividing this number by the total number of models. The shape of the sand patterns shows a high

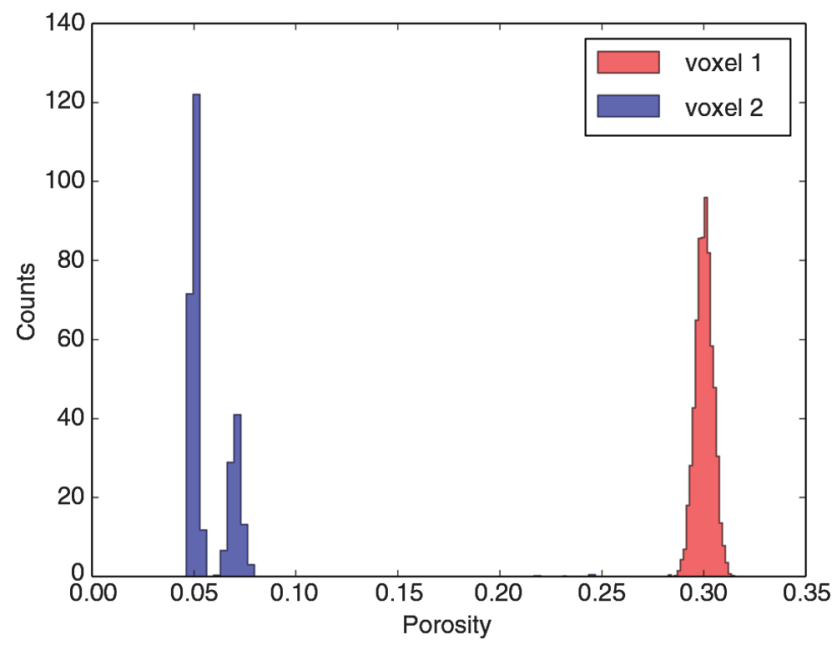

Figure 8. Histogram of porosity at two locations in the model obtained from the posterior collection of models.
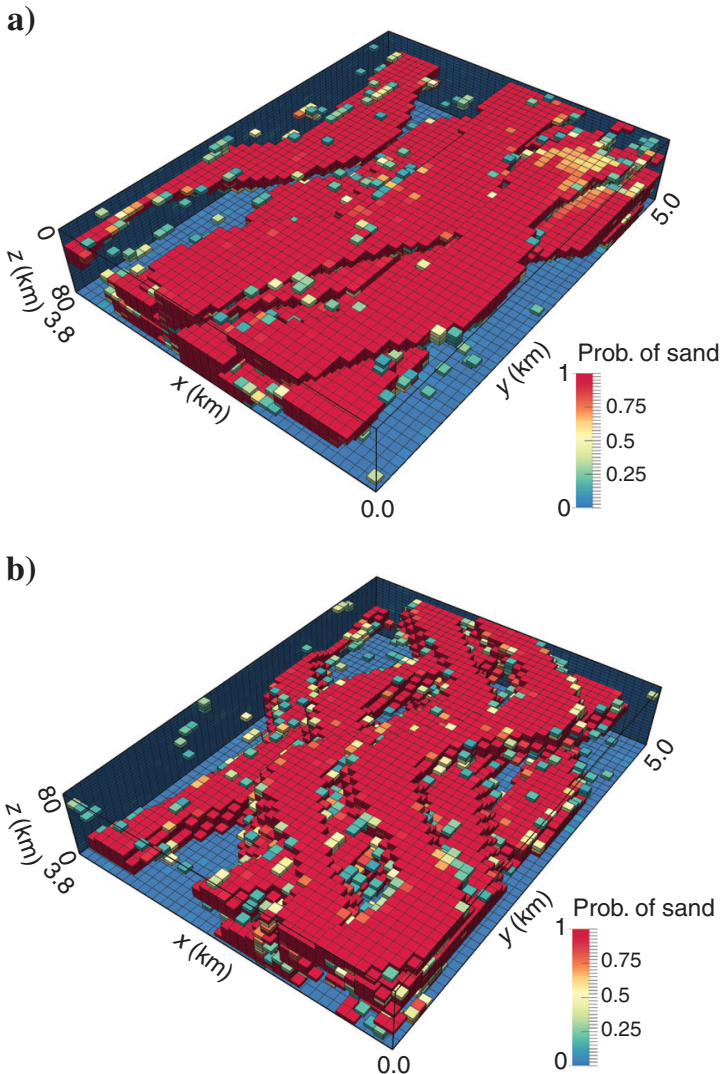

Figure 9. 3D map representing the probability of having sand as computed from the posterior collection. (a) View from above and (b) view from below. The values inside the volume have a lower threshold at 0.1 to allow us to see through the volume. degree of continuity in the channels. This feature is provided by the prior information as defined by the training image that, by means of geostatistical techniques, helps in maintaining spatial continuity in the structure of the models.

\section{CONCLUSION}

Analysis of target zones within a reservoir using Monte Carlo methods provides a comprehensive characterization of the posterior probability density, enabling us to perform uncertainty analysis. The McMC method grounded in the probabilistic approach to inverse problems can handle the nonlinearities within the forward model, such as the rock-physics model, and perform a simultaneous inversion allowing an unbiased estimate of uncertainties. We propose a methodology to fulfill these requirements that, using a parallelized version of the extended Metropolis algorithm, provides samples of the posterior distribution that represents a complete solution to the inverse problem. Samples from the prior distribution are provided by a geostatistical algorithm ensuring spatial continuity of the depositional structures designed in the prototype model. The use of informed priors reduces the search space thereby proposing only models consistent with a priori information.

This methodology integrates different kinds of information (geostatistical, petrophysical, seismic) into a single algorithm and is well suited to additional information input such as well log data, other measured geophysical data, and different forms of prior information.

We performed numerical experiments to test our methodology showing that it is possible to invert a relatively large number of parameters and provide advantages in terms of characterization of the posterior distribution. From synthetic measured seismic reflection data, we infer facies and porosity models and associated relative uncertainties. The database of solutions obtained is then queried to extract information we are interested in because it represents a complete solution to our inverse problem. Indeed, specific questions such as the probability of having a certain stratigraphic feature in the model, whose answers are difficult to obtain with traditional methods, are easily addressed within this framework.

\section{ACKNOWLEDGMENTS}

We thank DONG R\&D (Denmark) and Lloyd's Register/Scandpower for financial support. We are grateful to T. Fasnacht and two anonymous reviewers for their constructive comments on the manuscript.

\section{APPENDIX A ROCK PHYSICS}

The parameters used in this numerical experiment, following the Stanford VI setup, are critical porosity $\phi_{c}=0.38$, constant cement starting porosity $\phi_{b}=0.37$, coordination number $n=9$, P-wave calcite cement modulus $M_{c}=120.9 \mathrm{GPa}$, calcite cement shear modulus $G_{c}=32 \mathrm{GPa}$, and calcite cement Poisson's ratio $\nu_{c}=0.32$.

The Poisson's ratio of the solid phase of the rock $\nu_{s}$ is calculated from 


$$
\nu_{s}=\frac{1}{2} \frac{K_{s} / G_{s}-2 / 3}{K_{s} / G_{s}+1 / 3},
$$

whereas $\nu_{c}$ is assumed to be known.

The bulk modulus for fluid $K_{f}$ is computed using the Reuss lower bound (isostress) as

$$
K_{f}=\left[\sum_{i=1}^{2} \frac{S_{f(i)}}{K_{f(i)}}\right]^{-1} .
$$

The $K_{b}$ and $G_{b}$ from contact cement theory (Dvorkin and Nur, 1996) are calculated from

$$
K_{b}=\frac{n\left(1-\phi_{c}\right) M_{c} S_{n}}{6}
$$

and

$$
G_{b}=\frac{3 K_{b}}{5}+\frac{3 n\left(1-\phi_{c}\right) G_{c} S_{\tau}}{20}
$$

in which the various coefficients are given by

$$
\begin{aligned}
S_{n} & =A_{n} \alpha^{2}+B_{n} \alpha+C_{n} \\
A_{n} & =-0.024153 \Lambda_{n}^{-1.3646} \\
B_{n} & =0.20405 \Lambda_{n}^{-0.890008} \\
C_{n} & =0.00024649 \Lambda_{n}^{-1.9846} \\
S_{\tau} & =A_{\tau} \alpha^{2}+B_{\tau} \alpha+C_{\tau} \\
A_{\tau} & =-10^{-2}\left(2.26 \nu_{s}^{2}+2.07 \nu_{s}+2.3\right) \Lambda_{\tau}^{0.079 \nu_{s}^{2}+0.1754 \nu_{s}-1.342} \\
B_{\tau} & =\left(0.0573 \nu_{s}^{2}+0.0937 \nu_{s}+0.202\right) \Lambda_{\tau}^{0.0274 \nu_{s}^{2}+0.0529 \nu_{s}-0.8765} \\
C_{\tau} & =10^{-4}\left(9.654 \nu_{s}^{2}+4.945 \nu_{s}+3.1\right) \Lambda_{\tau}^{0.01867 \nu_{s}^{2}+0.4011 \nu_{s}-1.8186} \\
\Lambda_{n} & =\frac{2 G_{c}}{\pi G_{s}} \frac{\left(1-\nu_{s}\right)\left(1-\nu_{c}\right)}{1-2 \nu_{c}}, \Lambda_{\tau}=\frac{G_{c}}{\pi G_{s}} \\
\alpha & =\left[\frac{2\left(\phi_{c}-\phi_{b}\right)}{3\left(1-\phi_{c}\right)}\right]^{\frac{1}{2}} \cdot
\end{aligned}
$$

where $G_{s}$ and $\nu_{s}$ are the shear modulus and Poisson's ratio of the solid phase of the rock $G_{c}$ and $\nu_{c}$ are the shear modulus and the Poisson's ratio of the cement, and $\alpha$ is the ratio of the radius of the cement layer to the grain radius when cement is deposited evenly on the grain surface.

\section{REFERENCES}

Avseth, P., J. Dvorkin, G. Mavko, and J. Rykkje, 2000, Rock physics diagnostic of North Sea sands: Link between microstructure and seismic properties: Geophysical Research Letters, 27, 2761-2764, doi: 10.1029/ 1999GL008468.

Avseth, P., T. Mukerji, and G. Mavko, 2005, Quantitative seismic interpretation: Applying rock physics tools to reduce interpretation risk: Cambridge University Press.

Bosch, M., 2004, The optimization approach to lithological tomography: Combining seismic data and petrophysics for porosity prediction: Geophysics, 69, 1272-1282, doi: 10.1190/1.1801944.

Bosch, M., L. Cara, L. Rodrigues, A. Navarro, and M. Díaz, 2007, A Monte Carlo approach to the joint estimation of reservoir and elastic parameters from seismic amplitudes: Geophysics, 72, no. 6, O29-O39, doi: 10.1190/ 1.2783766 .

Bosch, M., T. Mukerji, and E. Gonzalez, 2010, Seismic inversion for reservoir properties combining statistical rock physics and geostatistics: A review: Geophysics, 75, no. 5, A165-A176, doi: 10.1190/1.3478209.

Brockwell, A. E., 2006, Parallel Markov chain Monte Carlo simulation by pre-fetching: Journal of Computational and Graphical Statistics, 15, 246261, doi: 10.1198/106186006X100579.

Cordua, K., T. Hansen, and K. Mosegaard, 2012, Monte Carlo full-waveform inversion of crosshole GPR data using multiple-point geostatistical a priori information: Geophysics, 72, no. 2, H19-H31, doi: 10.1190/ geo2011-0170.1

Doyen, P., 2007, Seismic reservoir characterization: An earth modelling perspective: EAGE Publications.

Dvorkin, J., M. Gutierrez, and D. Grana, 2014, Seismic reflections of rock properties: Cambridge University Press.

Dvorkin, J., and A. Nur, 1996, Elasticity of high porosity sandstones: Theory for two north sea data sets: Geophysics, 61, 1363-1370, doi: 10.1190/1 .1444059 .

Gardner, G., L. Gardner, and A. Gregory, 1974, Formation velocity and density - The diagnostic basics for stratigraphic traps: Geophysics, 39, 770-780, doi: 10.1190/1.1440465.

Gassmann, F., 1951, Über die elastizitt poroser medien: Veirteljahrsschrift der naturforschen: den Gesellschaft in Zürich, 961-23.

González, E., T. Mukerji, and G. Mavko, 2008, Seismic inversion combining rock physics and multiple-point geostatistics: Geophysics, 73, no. 1, R11R21, doi: $10.1190 / 1.2803748$.

Gouveia, W. P., and J. A. Scales, 1998, Bayesian seismic waveform inversion: Parameter estimation and uncertainty analysis: Journal of Geophysical Research, 103, 2759-2779, doi: 10.1029/97JB02933.

Grana, D., and E. Della Rossa, 2010, Probabilistic petrophysical-properties estimation integrating statistical rock physics with seismic inversion: Geophysics, 75, no. 3, O21-O37, doi: 10.1190/1.3386676.

Grana, D., T. Mukerji, J. Dvorkin, and G. Mavko, 2012, Stochastic inversion of facies from seismic data based on sequential simulations and probability perturbation method: Geophysics, 77, no. 4, M53-M72, doi: 10.1190/ geo2011-0417.1.

Guardiano, F., and M. Srivastava, 1993, Multivariate geostatistics: Beyond bivariate moments, in A. O. Soares, ed., Geostatistics Tróia '92, Kluwer Academic Publications, 133-144.

Hansen, T., K. Cordua, and K. Mosegaard, 2012, Inverse problems with nontrivial priors: Efficient solution through sequential Gibbs sampling: Computational Geosciences, 16, 593-611, doi: 10.1007/s10596-011-9271-1.

Hansen, T., K. Mosegaard, and K. Cordua, 2008, Using geostatistics to describe complex a priori information for inverse problems: Presented at Eighth International Geostatistics Congress, GEOSTATS, 2008, 329-338.

Hansen, T., K. Mosegaard, and K. Cordua, 2009, Reducing complexity of inverse problems using geostatistical priors: Presented at 15th Annual Conference of the International Association of Mathematical Geosciences (IAMG 09).

Hill, R., 1952, The elastic behaviour of a crystalline aggregate: Proceedings of the Physical Society, London, Section A, 65, 349, doi: 10.1088/0370$1298 / 65 / 5 / 307$

Journel, A., and C. J. Huijbregts, 1978, Mining geostatistics: Academic Press.

Karimi, O., H. Omre, and M. Mohammadzadeh, 2010, Bayesian closedskew Gaussian inversion of seismic AVO data for elastic material properties: Geophysics, 75, no. 1, R1-R11, doi: 10.1190/1.3299291.

Lange, K., J. Frydendall, K. Cordua, T. Hansen, Y. Melnikova, and K. Mosegaard, 2012, A frequency matching method: Solving inverse problems by use of geologically realistic prior information: Mathematical Geosciences, 44, 783-803, doi: 10.1007/s11004-012-9417-2.

Lee, J., and T. Mukerji, 2012, The Stanford VI-E reservoir: A synthetic data set for joint seismic-EM time-lapse monitoring algorithms: 25th Annual Report: Technical Report, Stanford Center for Reservoir Forecasting.

Mavko, G., T. Mukerji, and J. Dvorkin, 2009, The rock-physics handbook: Tools for seismic analysis of porous media: Cambridge University Press.

Mosegaard, K., 1998, Resolution analysis of general inverse problems through inverse Monte Carlo sampling: Inverse Problems, 14, 405-426, doi: $10.1088 / 0266-5611 / 14 / 3 / 004$

Mosegaard, K., 2006, Monte Carlo analysis of inverse problems: Doctoral thesis, University of Copenhagen, 61, ISBN 87-991228-0-4.

Mosegaard, K., 2012, Limits to nonlinear inversion, in K. Jónasson, ed., Applied parallel and scientific computing, Springer, Lecture Notes in Computer Science Series, volume 7133, 11-21.

Mosegaard, K., and A. Tarantola, 1995, Monte Carlo sampling of solutions to inverse problems: Journal of Geophysical Research, 100, 1243112447, doi: $10.1029 / 94 J B 03097$.

Mosegaard, K., and A. Tarantola, 2002, Probabilistic approach to inverse problems, in W. Lee, P. Jennings, C. Kisslinger, and H. Kanamori, eds., International handbook of earthquake and engineering seismology: Academic Press, 237-265. 
Remy, N. A. Boucher, and J. Wu, 2009, Applied geostatistics with SGEMS: A user's guide: Cambridge University Press.

Strebelle, S., 2002, Conditional simulation of complex geological structures using multiple-point statistics: Mathematical Geology, 34, 1-21, doi: 10 1023/A: 1014009426274.

Tarantola, A., 2005, Inverse problem theory and model parameter estimation: SIAM.

Ulvmoen, M., and H. Omre, 2010, Improved resolution in Bayesian lithology/fluid inversion from prestack seismic data and well observations-
Part 1: Methodology: Geophysics, 75, no. 2, R21-R35, doi: 10.1190/1 .3294570 .

Ulvmoen, M., H. Omre, and A. Buland, 2010, Improved resolution in Bayesian lithology/fluid inversion from prestack seismic data and well observations - Part 2: Real case study: Geophysics, 75, no. 2, B73-B82, doi: 10.1190/1.3335332.

Woodward, M., D. Nichols, O. Zdraveva, P. Whitfield, and T. Johns, 2008, A decade of tomography: Geophysics, 73, no. 5, VE5-VE11, doi: 10.1190/1 .2969907 . 